\title{
STAGING CO-DESIGN FOR A CIRCULAR ECONOMY
}

\author{
Pedersen, Signe; Clausen, Christian
}

Aalborg University

\begin{abstract}
In recent years major companies such as Philips, H\&M and Google have adopted a circular economy agenda to promote sustainability. Design consultancies such as IDEO has developed and promoted a circular design guide to help companies in this endeavour. However, designing for a circular economy often require design and reconfiguration of entire value chains - making the transition towards a circular economy rather difficult. In this paper we analyse a development project from the Danish island of Bornholm to investigate how to align diverse actors across the value chain in a process of co-creating systems for a circular economy. We combine design, value chain considerations and circular economy mindsets to informing negotiations of concerns among actors in the value chain.

Such strategical navigation might involve:

1. Staging initial spaces for dialogue with central actors from the value chain and initiates a process of mapping out their concerns

2. Staging a re-alignment space where the diverse actors can meet and interact to discuss and negotiate their concerns and their roles in the network

3. Design and enactment of a number of objects to faciliate negotiations
\end{abstract}

Keywords: Circular economy, Business models and considerations, Participatory design, Re-alignment, Negotiation

Contact:

Pedersen, Signe

Aalborg University

Development \& Planning

Denmark

sigpe@plan.aau.dk

Cite this article: Pedersen, S., Clausen, C. (2019) 'Staging Co-Design for a Circular Economy', in Proceedings of the 22nd International Conference on Engineering Design (ICED19), Delft, The Netherlands, 5-8 August 2019. DOI:10.1017/dsi.2019.344 


\section{INTRODUCTION}

Major leading organisations around the world such as Philips, Google and H\&M have adopted a circular economy agenda to promote global sustainable transitions. Design thinking has been suggested as a general approach to enhance sustainable business modelling processes and design for a circular economy (Geissdoerfer, Bocken and Hultink, 2016). For instance, IDEO (one of the world's leading design thinking consultancies) has developed a Circular Design Guide (IDEO, 2016) in collaboration with the Ellen Macarthur Foundation to help organisations re-design products, services and business models in support of the development of a circular economy. The design thinking inspired methods presented in this Circular Design Guide is highly firm-centric, as they offer checklists for an organisation to consider when they decide to embark on a journey towards thinking and designing for a circular economy.

However, when designing solutions for a circular economy, more often than not, this entails (re)designing not only products and services but also entire systems, which are dependent on numerous other stakeholders from the value chain. In these situations, co-creation across the value chain is necessary to ensure sustainable solutions, and this is where the firm-centric design thinking methods falls short.

\section{CURRENT UNDERSTANDING}

\subsection{Transition towards a circular economy requires collaboration}

The dominant concept within circular economy includes a substitution of the traditional linear model of make-use-dispose to ensure that we keep resources in use for as long as possible by qualified maintenance, re-use, refurbishment and re-cycling (Ellen MacArthur Foundation, 2013). Many examples of such projects take a starting point in a sourcing perspective from larger organisations' point of view using their scale and influence to pressure their suppliers to substitute processes or materials in components, while others use more cooperative strategies across the supply chain (Forman, 2004). For instance, several tools for mapping potential value such as the "multiplestakeholder value perspective' has been introduced (Bocken, Rana and Short, 2015), and open innovation literature promotes co-creation of value together with customers. Both of these approaches are highly firm-centric and presuppose that the stakeholders have defined value propositions which can be identified and mapped. However, when it comes to cases of jointly co-creating new business models and product- service systems the complexity increases as the involved stakeholders often has multiple agendas and interests, which cannot be easily mapped in advance. Christensen et al. (2017) move the perspective from the single company to take a cross firm perspective and study co-creative activities spanning supplier - firm networks as they have examined the challenges (which they treat as 'wicked problems') associated with the creation of joint innovation across the value chain. In their study of eight Danish cases of supplier driven innovation, they identified a number of collaborative spaces for jointly specifying solutions and expanding innovative solution spaces. But, while these approaches may point at the phenomena and emergence of collaborative approaches to design and innovation across actors such as users and organisations, it is less clear how to create or support the development of such collective spaces of collaboration. But, a key consideration is to point at a number of techniques from the co-design design approaches that may encourage dialogue among the participants through tangible means (Christensen, Munksgaard and Bang, 2017).

\subsection{Designing for a circular economy}

A wide range of tools and methodologies have been developed from a design perspective to support decisions in designing Product Service Systems, (PSS) which have some similarities to circular economy as it also requires a systemic approach considering all elements such as products, services and a network of actors simultaneously. But this methodology mainly focusses on creation of decision support (Ceschin and Gaziulusoy, 2016) and not on the wider process of co-designing these networks. The design thinking community, with IDEO in a leading role, are promoting tools, methods and an overall design process to facilitate co-creation activities. Andrews (2015) suggests that designers should take on a leading role in designing for a circular economy by designing for disassembly and waste cycling changing the perception of value. But while their visualisations of the design process are indeed very communicative and easy to follow, none of the proposed tools and frameworks from 
design thinking or sustainable business thinking offer any perspectives on how co-creation might be seen as a 'negotiation' between stakeholders in order to align their interests. Wetter-edman, Vink, and Blomkvist (2017) warn against the general applicability of generalised design thinking approaches and point at their lack of attention to situational aspects of design and the embodied nature of acting. Instead they point at the need to focus on, where disruption of current institutionalised practices may occur. Consequently, they suggest to stage situations, where actors may sense contradictions, experience conflicts, and in this way trigger inquiry and question taken for granted assumptions. Contradictions and conflicts usually require negotiation in order to create re-alignment of interests (Carlile, 2002; Clausen and Yoshinaka, 2007; Iversen, Halskov and Leong, 2012). This collaborative approach challenges the traditional $\mathrm{CE}$ principles of maintaining, reusing, refurbishing and recycling as the issue is no longer only a question of which predefined principle or strategy to use but rather how to stage negotiations between actors from the value chain where strategic choices may not easily align with CE principles. And while design thinking falls short in terms of providing a framework for negotiating value in systems design across the value chain (Kimbell 2011), other design approaches such as participatory design promotes the importance of staging and facilitating negotiations between a multiplicity of stakeholders (Iversen, Halskov, and Leong 2012).

\subsubsection{Staging negotiations}

Within participatory design, the designer, in contrast to for instance Design Thinking, is not seen as the central creative figure who comes up with solutions based on customer insights. Instead, the designer is an orchestrator who stages negotiations - for instance in workshops using mock-ups and prototypes as knowledge objects in order to frame particular negotiations (Iversen, Halskov, and Leong 2012). Furthermore, participatory design and participatory innovation practitioners and scholars highlights the direct involvement and influence of not only users but the broader array of actors from the value chain throughout the design process (Buur and Matthews, 2008). But, while participatory design provides a practical and inclusive approach to collaboration, it does not explicitly occupy itself with the creation of networks, ecologies and systems constituted by assemblies of actors and objects established over longer periods of time as is the case in innovation (Björgvinsson et al., 2012). The involvement of many actors in the design process makes design not only a process of change but also a political process where different concerns and perspectives are to be taken into account and negotiated across the diverse actors (Bucciarelli, 1994; Simonsen and Robertson, 2012; Halskov and Hansen, 2014). Design becomes a question of establishing the conditions for bringing together actors to negotiate (meaning to sketch, prototype, visualise knowledge and ideas) in e.g. meetings or workshops around diverse intermediary objects (Boujut and Blanco, 2003; Vinck, 2012), and apply design games or other facilitation techniques to frame the negotiations. These activities of continued support of engaging participants in processes of dialogue and design is what can be termed infrastructuring (Bjørgvinsson et al., 2012) or participatory infrastructuring (Bødker, Dindler and Iversen, 2017)

In order to understand the possibilities for implementing a circular economy concept and contribute to the management of the innovative processes needed, we have identified a gap in current research concerning the negotiations and realignment of actors and objects across the value chain. In order to address this shortcoming, we suggest to combine the design approach of infrastructuring with the theoretical framework provided by Actor Network Theory (ANT) to shed light on negotiations and translation of knowledge, aligning actors and building collaborative networks in support of designing for a circular economy (Björgvinsson, Ehn and Hillgren, 2012).

\section{RESEARCH QUESTION}

We want to shed light on the efforts of co-design for a circular economy together with a range of actors in the value chain. Based on this, we state the following research question: How to stage negotiations across diverse actors of the value chain when co-designing systems for a circular economy.

\section{RESEARCH DESIGN}

This particular case study was part of a larger research and development project funded by the Danish Industry Foundation. The aim of this research project was to study 20 cases of Danish companies embarking on a journey towards a transition to a circular economy. Each company and thus each case 
is unique and thus the transition process is initiated based on situated local value chain structures and motivations. One of the authors of this paper, played an active role as designer and facilitator in the particular case study subject to investigation and analysis in this paper along the principles of action research (Kagan, Burton and Siddiquee, 2006).

The central company in this specific case project was a professional laundry operator at the Danish island of Bornholm. The motivation of the laundry operator was a desire to optimize the laundry services system for his public and private clients with a particular focus on the only public hospital situated on the island. With a starting point in the value chain, the author, during approximately one year, participated actively in the project by engaging relevant actors from the value chain using roll the snowball techniques, conducting multiple interviews with each actor (see table 1). Furthermore, the author did multiple fieldwork studies at the local hospital through participant observation (DeWalt, DeWalt R. and Wayland, 1998) where patients, nurses, cleaning staff and actors from facilities management was observed and interviewed while performing their daily tasks. Based on these activities a workshop involving most of the value chain was staged and facilitated in order to allow for the participant to negotiate a shared goal and strategy for the implementation of the project.

Table 1: Overview of research and design activities

\begin{tabular}{|c|c|c|}
\hline Activity & Participating actors & Purpose \\
\hline Workshop 1 & $\begin{array}{l}\text { Laundry service provider, hospital facilities } \\
\text { managers, representative from textile company, } \\
\text { Bornholm public water utility }\end{array}$ & $\begin{array}{l}\text { Allowing for the actors of the value chain to } \\
\text { meet and discuss/negotiate common grounds }\end{array}$ \\
\hline Workshop 2 & $\begin{array}{l}\text { Laundry service provider, hospital facilities } \\
\text { managers, }\end{array}$ & Negotiate the object of design (focus areas) \\
\hline Interviews & $\begin{array}{l}\text { Laundry service provider, hospital facilities } \\
\text { managers, laundry machine manufacturer }\end{array}$ & $\begin{array}{l}\text { Allowing each actor from the value chain to } \\
\text { voice interest and concerns }\end{array}$ \\
\hline Field work & $\begin{array}{l}\text { Researcher following the textiles on their way } \\
\text { through the hospital }\end{array}$ & $\begin{array}{l}\text { Understanding the concerns of cleaning } \\
\text { staff, patients and nursing staff }\end{array}$ \\
\hline Continuously & $\begin{array}{l}\text { Researcher in iterative process with key value } \\
\text { chain actors }\end{array}$ & $\begin{array}{l}\text { Translating findings and insights into flow } \\
\text { charts, problem statements, user journeys, } \\
\text { PP presentations etc. }\end{array}$ \\
\hline
\end{tabular}

The action research serves as an inductive case study to build understanding in relation to the research question aiming to analyse how diverse actors with somewhat contradicting positions through a creative co-design project is able to create alignment and collaboration through negotiation. Based on the above-mentioned actions the one author kept a log book and produced a number of transcriptions of interviews which together with a variety of created design objects became the basis for the analytical research process. With respect to the data analysis, the case narrative was written based on an abductive analysis (Timmermans and Tavory, 2012) carried out by both authors in order to ensure a more independent reflection. This led us to iteratively revisit our empirical data using sensitizing devices such as the concepts of temporary negotiation spaces and knowledge objects (Pedersen and Clausen, 2017).

\section{SUSTAINABLE PROFESSIONAL LAUNDRY SERVICES AT BORNHOLM}

As mentioned, this particular case study was part of a larger research and development project funded by the Danish Industry Foundation involving 20 companies in Denmark. One of the 20 companies, which will serve as 20 individual exemplifying case studies, is an industrial laundry service provider situated on the island of Bornholm. Bornholm is interesting in relation to sustainability and transition towards clean energy initiatives, as its size is just right for serving as a test hub for new initiatives. The island is small enough to provide a rather tightly knitted community and large enough to have attracted sizable public and private actors such as: public infrastructure including a hospital and a public utility provider, world-leading companies such as a manufacturer of turn-key solutions and machinery for professional industrial laundries who is organized into five worldwide business regions and three technology centers: Washroom Technology, Finishing Technology and Material Handling. 


\subsection{Project framing in the outset: TCO as main concern?}

As the goal of the research project was to enable companies to be part of a transition towards a circular economy, this was also the frame and agenda which the industrial laundry initially signed up for. The industrial laundry is a front runner in terms of transforming their laundry services to meet a sustainable future. Around project kick-off the researchers had a couple of meetings with the CEO of the industrial laundry as well as the CEO of the manufacturer of turn-key solutions for industrial laundries whom also expressed interest in joining the research project. The latter was quite focused on TCO (total cost of ownership) considerations as some of his public customers (mainly hospitals) had expressed an interest in including such calculations as part of ongoing discussions about promoting more sustainable public procurement structures. The CEO of the industrial laundry experienced similar interests from his customers and hence also saw the potential of looking into TCO considerations potentially offering main customers (local hotels and the public hospital) an opportunity to buy 'clean sheets' rather than 'the wash of e.g. 1000 sheets'. This would mean that the industrial laundry and not their customers would be the ones owning the products (sheets and towels). At this point in time the primary researcher needed to shift her attention to work on another research project which meant that a new researcher with a background in design and innovation was introduced to the project. The new researcher approached the project in a new way seeking to stage co-creation amongst the actors in the value chain and initiated a process of negotiations and infrastructuring.

\subsection{Re-framing the project: How can CE support saving lives?}

In order to get up to speed, the new researcher had meetings with both the industrial laundry, one of their customers (a local hotel) and the manufacturer of laundry equipment. She had prepared a very simple design game to play with each of the stakeholders during the meetings, asking them to rank a number of cards illustrating and representing different aspects of circular economy considerations and how they could be translated into value for the various actors. Examples where: trust, economy, service, customers, sustainability etc. which served as the starting point and a way to frame and guide the conversations. And during the discussions new post-its were added with new insights and concerns voiced by the participants.

The professional laundry's CEO explained about previous initiatives related to optimizing energy savings and cleaning up the wastewater, but he did not directly mention any interest in TCO considerations. The researcher expected that surely the CEO of the laundry equipment manufacturer would mention and promote the TCO agenda, and was puzzled when he also did not call out TCO as a concern. From her colleagues the researcher had been briefed that the project was about TCO concerns, and when she asked about it, the CEO explained, that while the project had been paused, he had done some thinking and concluded, that it would be extremely challenging for him to turn his company from being a manufacturer of products to being a service provider. He told, that he already had challenges finding skilled labour and blamed mothers in general for pushing their sons towards universities rather than training to become craftsmen. Thus, he would only consider this encompassing strategic move if a major customer 'the size and scale of China' would require him to do so. To this worldwide company a customer and a research project in small Denmark would not be incentive enough for him to shift focus. This illustrates that this CEO is very much concerned with the interests of his customers, however, he cannot make major changes in his business model until a sizable customer asks for it.

After the initial meetings a picture was forming that the common denominator for both CEOs was a wish to learn more about the other actors in the value chain and make an even bigger environmental impact by strengthening and potentially re-configuring the relationships with the other actors. In particular, both the CEO of the washing equipment manufacturer as well as the CEO of the professional laundry expressed an interest in getting to know the concerns of the downstream actors in the value chain and thus allow these actors concern to inform the further framing of the project. The publicly owned hospital was of particular interest for the two CEOs, as Bornholm's hospital is part of a larger system constituting the public hospital sector in Denmark (and has special links to the main hospitals in the Copenhagen capital area). Hence the researcher contacted the hospital and set up a meeting with the leading facilities managers as they were the immediate customers and in charge of the public procurement process related to washing-services. At this meeting the facilities managers were introduced to the project setup, encouraged to play the same design game as the rest of the actors, and by the end of the meeting also invited to participate as a central actor in the research project. They 
accepted the invitation as they were eager to understand the concern of their 'customers', the patients, and provide a better service and experience to support the overall concern of the hospital which is to save lives and increase patients' chances of an early recovery (see figure 1).

Hence, we begin to see a desire and a concern among all the actors in the value chain to understand the concerns of their customers, in this case facilities managers and patients, which are all situated at the local public hospital. The overall concern of the hospital is to 'save lives' and enable a speedy recovery, and as the rest of the actors in the value chain are eager to support the concern of the hospital, all of the value chain actors are motivated to aligning their efforts in this direction. So, at this moment there is alignment in terms of wanting to promote circular economy related efforts AND in terms of supporting the goal of saving lives at the hospital.

\subsection{Unfolding the relationship between 'saving lives' and hospital textiles}

But how does facilities managers, laundries and manufacturers of laundry machinery support saving lives at the hospital? The concern of 'saving lives' needs to be translated into something more tangible that the actors in the value chain can support - and which also promotes a circular mindset. All actors were eager to know the aspirations and the matters of concern of their customers and thus also of the actors at the hospital. However, none of them really knew where to begin. To kick-start this process, the researcher/designer offered to conduct field work at the hospital following the textiles on their way from being delivered by the laundry, distributed to the wards, worn by the staff and patients and being sent back to the laundry. Furthermore, she offered to talk to patients and staff about how they see the clean and comfortable textiles support the concern of saving lives and improve the health of the patients. Numerous studies suggest that textiles and especially the patient's clothing is part of a 'healing environment' (Topo and Iltanen-Tähkävuori, 2010) and thus it seemed fair to link these two together. To make these rather big and complex questions and concerns more concrete and tangible, the designer synthesized the insights from the initial meetings with the laundry CEO, the washing equipment manufacturer and the facilities managers into a number of themes which could be investigated by posing questions at the hospital related to for instance: how the textiles smell after being washed, how comfortable and wearable is the fabric, are there enough patient shirts available in the right sizes at the right time etc. With these questions as the starting point, the designer arranged to talk with patients and staff taking an exploratory approach in terms of wanting to learn from patients and staff about their experiences and interactions with the hospital clothes, the bed linen, the towels etc. Hence, the space spanning the fieldwork activities was not tightly staged but instead rather open allowing patients and staff to bring forward any concerns related to the hospital textiles. Furthermore, the researcher wanted to use the opportunity to learn from the cleaning staff and others from facilities management about their working routines in terms of the logistics related to the textiles.

During her visits to the hospital she engaged with at least 5 patients, 2 nursing staff and 2 cleaning ladies, the employee at the linen-room. And two reoccurring themes kept emerging: 1) The patients all complained about the pillows being too hard and bulky. As one of the patients stated: 'it's like lying on a cannon ball'. Not only the patients but also the staff had picked up on the need for better pillows as they shared how they would sometimes try out 2-3 different pillows for the patients before they found one that provided decent comfort, and 2) The staff which had been at the hospital for many years had noticed a change in the service-level from the linen-room staff. According to the staff, the service from the person in charge of the linen room (who receives the clean textiles from the laundry and makes sure that it is distributed to the wards at the hospital and who hands out clean clothes to the hospital staff) had decreased since 'back in the days' where one dedicated person employed by the hospital was working there full time - as supposed to the current situation where changing staff employed by the professional laundry works there half a day at a time.

\subsection{Translating concerns into knowledge objects and redefining the value chain}

The interviews and fieldwork conducted might be seen as so-called front stage activities where actors from the value chain is involved in negotiations and in expressing their concerns. At the same time, 'backstage activities' are taking place to build knowledge objects that help to represent the current frames or overall concerns but also to mediate and serve as intermediary objects between diverse actors. An example of such an activity is when the researcher translated voiced concerns and inscribed these into different kinds of materiality such as flow charts and illustrations of user-statements to be used as intermediary objects in further negotiations among actors in the value chain (see Figure 2). These were sent by email to the key actors in the project to illustrate the preliminary insights and to 
give them an idea of what was the basis of the future negotiations. As the CEO of the washing equipment manufacturer was quite busy and the same time reluctant in making any changes in his business model the key actors was at this point: the CEO of the laundry, a local representative from a Danish textile company currently collaborating with the professional laundry, and the facilities managers at the hospital. The representative from the textile company was involved as his company had invented a new type of weaving that would prolong the lifetime of their textiles while still being comfortable to wear. This new textile would also benefit the professional laundry as it required less drying time and thus reduced energy consumption.

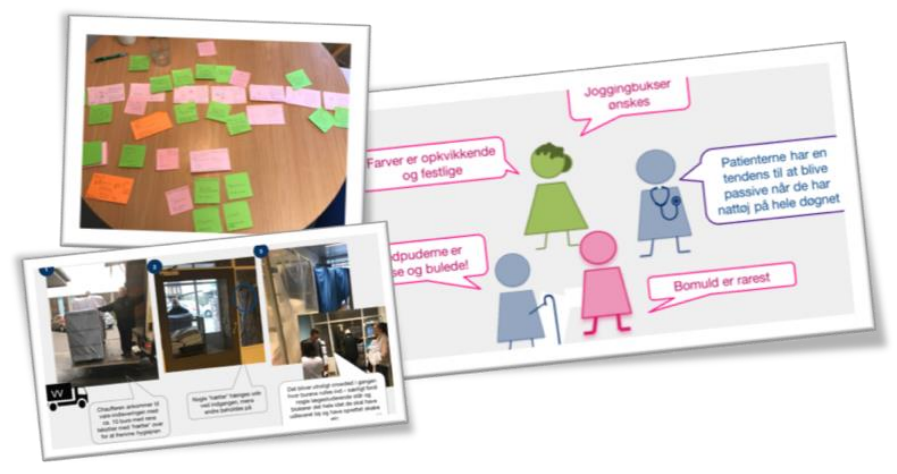

Figure 2: Examples of intermediary objects

\subsection{Staging a workshop for negotiation across the value chain}

At this stage, some concerns were identified, but the project scope and what is to be the object of design was still rather open or 'wicked'. So, next step was to stage a workshop where multiple actors from the value chain would be able to participate in 'collective sensemaking' (Christensen, Munksgaard and Bang, 2017) around the identified concerns being 'saving lives' and designing for a circular economy. The aim was, that the negotiations done during collective sensemaking would result in the identification of a more tangible object of design which where to form the basis of the next phase in the project related to co-designing solutions through network (re)alignment. At this point, not everybody from the value chain had met each other, and thus this was the perfect opportunity to do so and to see what (if anything) they had in common and could agree on working together to accomplish. The CEO of the professional laundry, the representative from the textile company, two facilities managers from the public hospital and two representatives from the public utilities' organisation participated in the workshop. Unfortunately, the CEO of the manufacturing company was not able to attend the workshop due to other engagements, but otherwise most of the value chain was represented. The program for the workshop was centred around a visual illustration of the value chain (Figure 4 left picture), and the participants were given post'its to write down their matters of concern as well as their aspirations in terms of participating in the research project. The researcher/designer staged and facilitated the workshop based on the initial engagements with the invited actors. She also represented the patients and the staff whom she had encountered at the hospital - both by speaking on their behalf and by including the produced illustrations of the user-statements in the discussions.
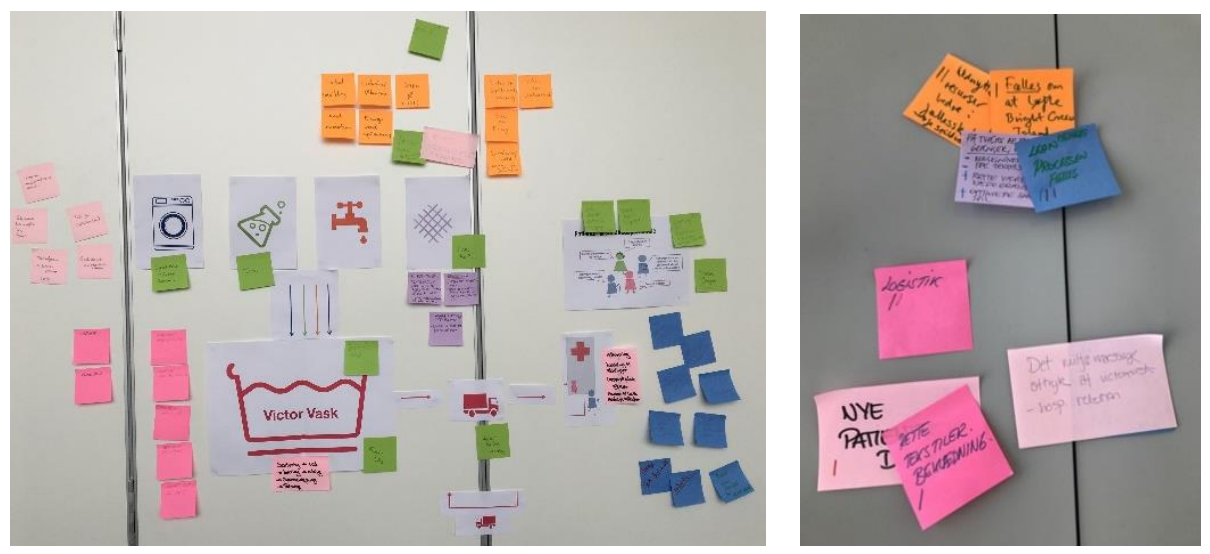

Figure 4: The value chain with actor's post'its 
Every participant wrote down their interests and concerns and presented it to each other, and as it turned out, what all actors presented was a desire to work together towards Bornholm becoming a 'bright green island' which was the political strategy for the island. Committing to this strategy would provide the organisations with branding opportunities as well as a possibility of applying for public funding to support this political endeavour. Hence, the bright green island strategy turned out to be a crucial 'object' for creating alignment among the actors across the value chain.

Furthermore, the user-statements, representing concerns from patients and hospital staff, served as a translation of 'saving lives', and thus provided the framing for the solution space. This meant, that by the end of the workshop, the participants expressed a shared desire and a vision to work together for creating a sustainable product service system centred around comfortable pillows and patient clothing.

\subsection{Immediate outcome}

After the workshop a core-group of actors formed involving the CEO of the laundry, the hospital facilities managers, and the representative from the textile company who had changed job and was now employed by the professional laundry. At this meeting a final negotiation took place in terms of the project focus. As patients clothing would require design skills and competencies not available amongst the current actors in the value chain, the core-group chose to go on exploring the opportunity of either developing or buying new comfortable pillows. The criteria for these pillows were that they wouldn't need pillow cases, that they could be washed and dried at low temperatures without using too much washing detergent in the process, and that they had a filling that would not deteriorate during wash. What still needed to be discussed was whom should invest in these pillows, the hospital or the professional laundry. As it turned out, there was to be a national public procurement process related to buying new pillows for hospitals all over Denmark, and Bornholm's hospital chose to participate in this, taking with them the insights learned and the concerns shared as part of the research project.

Furthermore, the facilities managers inscribed lessons from the flow charts and the patients- and staff statements into the upcoming public tender on laundry services which also involved logistics. Thus, the illustrations of patient statements and flow charts of the journey of the textiles was actively used in new negotiations related to services and to who was to be in charge of which processes.

\section{DISCUSSION}

This case illustrates how traditional design thinking efforts falls short in complex systems involving actors from the entire value chain. As it turned out, the majority of the work consisted of staging negotiations to identify the object of design - which turned out to be a combination of products, services and logistics which entails a reconfiguration of the value chain. Thus, in line with Ceschin (2014), the object of design is the network of the value chain and not a product or service in itself. So, rather than the designer being a 'creative' element designing products, the designer/researcher's role has become a networker with the role of enabling negotiations about circular economy. In this process a key attention should be with the concerns of the actors having different positions concerning the creation and capture of value in order to seek re-alignment in the network. The above co-design process was enabled through establishing a new supportive infrastructure (Bødker, Dindler and Iversen, 2017) where each actor is enabled to define their role in the network of the value chain by relating themselves to circular economy aspects and user-concerns. In this sense, circular economy becomes a sensitising device which contributes to frame the process and drive the identification of possibilities for alignment (the solution space). And this device is set into play as part of organising the strategic staging of the negotiation between the actors.

Based on our analysis of the above experimental case, we can suggest the following elements with which a designer may strategically navigate by staging a realignment of a value chain in order to enable co-design for a circular economy:

1. The designer stages spaces for dialogue with central actors from the value chain and initiates a process of mapping out their concerns

2. The designer stages a space where the diverse actors can meet and interact to discuss and negotiate their concerns and their roles in the network in a process of (re)alignment

3. As a key enabler of the above elements, the designer produces and enacts a number of objects. Firstly, the designer uses circular economy related objects such as cards representing different aspects of circular economy considerations to frame the initial dialogue of the actors in a mapping process aiming at identifying actor's concerns. Originally focus was on TCO, but as it turned out, all the 
central actors in the value chain was interested in getting to know their customers better - and all of these customers was situated at the local hospital. On this ground the facilities managers where invited to participate and the overall concern of the hospital, 'saving lives', framed the following discussions and thus also the solution space, which is seen as highly 'wicked' (Christiansen and Gasparin, 2017) and remained rather open until the very end of the project period.

Secondly, a workshop was stages where central actors from the value chain was invited. The negotiations taking place in this space led to a side-lining of the manufacturing company as its CEO did not share the same enthusiasm for collaborating in bright green island related activities. Thus, we see that the concerns of the actors (as well as the actors) themselves are shifting and open for negotiation. But, the case also shows, that a consequence of following the concerns the actors may be, that key principles and solutions associated with circular economy are not being implemented if it does not align with their interests. In this sense, while the staging approach may perform an important realignment of the value chain by reframing value from a new perspective on users, it is no "silver bullet' that solve all problems. However, there is no way an implementation of CE principles can take place if key actors are not getting interested in realigning business models across the value chain, so we still see co-design as a promising approach to enable such a translation of interest.

In order to enable this translation process a number of situated objects was produced and enacted. The Bright green island-strategy became a central object to become enacted, but also the initial design games, flow charts and user-statements mediated between actors and then enabled the recruitment process by serving as intermediary objects used actively by the designer to stage the negotiations of concerns. Inscribed in these objects was also the concerns of the patients and staff which was not invited to participate in the workshop at that stage. Thus, we see how the results of the initial mapping is inscribed in the staging of the workshop and used actively in the facilitation of the negotiations. The outcome of the workshop was a solution space of opportunities identified by the actors in the value chain themselves, namely new pillows and an improved logistics system to handle the flow of textiles and prolong the product life of the new pillows.

\section{CONTRIBUTION}

In this article we have tried to integrate perspectives from design, value chains and circular economy and find that even though this was a project about circular economy, the researcher/designer did not immediately seek to identify further potentials for energy savings (or to prolong the product life as part of a circular economy agenda) but rather to strategically navigate by re-organising the relationships and the collaboration between the actors in the value chain so that they together would negotiate concerns and come up with new solutions to meet the potentials for closing circular loops. In this way the paper offers a new approach to enable co-design for a circular economy based on strategic navigation and staging of the realignment of value chains

\section{REFERENCES}

Andrews, D. (2015), “The circular economy, design thinking and education for sustainability”, Local Economy, Vol. 30 No. 3, pp. 305-315. http://dx.doi.org/10.1177/0269094215578226.

Björgvinsson, E.B., Ehn, P. and Hillgren, P.-A. (2012), "Design Things and Design Thinking: Contemporary Participatory Design Challenges", Design Issues, Vol. 28 No. 3, pp. 101-116.

Bocken, N.M.P., Rana, P. and Short, S.W. (2015), "Value mapping for sustainable business thinking", Journal of Industrial and Production Engineering, Vol. 32 No. 1, pp. 67-81. Taylor \& Francis, http://dx.doi.org/10.1080/21681015.2014.1000399.

Bødker, S., Dindler, C. and Iversen, O.S. (2017), "Tying Knots: Participatory Infrastructuring at Work", Computer Supported Cooperative Work: CSCW: An International Journal, Vol. 26 No. 1-2, pp. 245-273. Computer Supported Cooperative Work (CSCW), http://dx.doi.org/10.1007/s10606-017-9268-y.

Boujut, J.-F. and Blanco, E. (2003), "Intermediary Objects as a mean to foster Co-operation", Computer Supported Cooperative Work, Vol. 12, pp. 205-219.

Bucciarelli, L.L. (1994), Designing Engineers, MIT Press.

Buur, J. and Matthews, B. (2008), "Participatory Innovation", International Journal of Innovation Management, Vol. 12 No. 3, pp. 255-273. http://dx.doi.org/10.1142/S1363919608001996.

Carlile, P.R. (2002), "A Pragmatic View of Knowledge and Boundaries : Boundary Objects in New Product Development", Organization Science, Vol. 13 No. 4, pp. 442-455.

Ceschin, F. (2014), "How the Design of Socio-technical Experiments Can Enable Radical Changes for Sustainability", International Journal of Design, Vol. 8 No. 3, pp. 1-21. 
Ceschin, F. and Gaziulusoy, I. (2016), "Evolution of design for sustainability: From product design to design for system innovations and transitions", Design Studies, Vol. 47, pp. 118-163. http://dx.doi.org/10.1016/j.destud.2016.09.002.

Christensen, P.R., Munksgaard, K.B. and Bang, A.L. (2017), “The wicked problems of supplier-driven innovation”, Journal of Business and Industrial Marketing, Vol. 32 No. 6, pp. 836-847. http://dx.doi.org/10.1108/JBIM-06-2015-0110.

Christiansen, J.K. and Gasparin, M. (2017), "How experiments in the fuzzy front end using prototyping generates new options", Journal of Experimental Innovation, Vol. 1 No. 1, http://dx.doi.org/10.23726/cij.2017.463.

Clausen, C. and Yoshinaka, Y. (2007), "Staging socio-technical spaces: translating across boundaries in design", Journal of Design Research, Vol. 6 No. 1/2, pp. 61-78. http://dx.doi.org/10.1504/JDR.2007.015563.

DeWalt, K.M., DeWalt, R.B. and Wayland, C.B. (1998), "Participant Observation", Handbook of Methods in Cultural Anthropology, pp. 259-299.

Ellen MacArthur Foundation (2013), “Towards the Circular Economy”, Ellen MacArthur Foundation, Vol. 1, pp. 1-96.

Forman, M. (2004), “Organising Environmental Supply Chain Management”, Greener Management International, Vol. 45 No. 45, pp. 43-62. http://dx.doi.org/10.9774/GLEAF.3062.2004.sp.00005.

Geissdoerfer, M., Bocken, N.M.P. and Hultink, E.J. (2016), "Design thinking to enhance the sustainable business modelling process - A workshop based on a value mapping process", Journal of Cleaner Production, Vol. 135, pp. 1218-1232. Elsevier Ltd, http://dx.doi.org/10.1016/j.jclepro.2016.07.020.

Halskov, K. and Hansen, N.B. (2014), "The diversity of participatory design research practice at PDC 20022012”, International Journal of Human-Computer Studies, Vol. 74, pp. 81-92. Elsevier.

IDEO, E. M. F. + (2016) The Circular Design Guide. Available at: https://www.circulardesignguide.com.

Iversen, O.S., Halskov, K. and Leong, T.W. (2012), "Values-led participatory design”, CoDesign, Vol. 8 No. 2 3, pp. 87-103. http://dx.doi.org/10.1145/2399016.2399087.

Kagan, C., Burton, M. and Siddiquee, A. (2006), "Participatory design and action research: Identical twins or synergetic pair", Handbook of qualitative research methods in psychology, Vol. i No. 5, pp. 93-96. http://dx.doi.org/10.1007/s11213-009-9145-9.

Kimbell, L. (2011), “Rethinking Design Thinking: Part I”, Design and Culture, Vol. 3 No. 3, pp. $285-306$.

Pedersen, S. and Clausen, C. (2017), "Staging Collaborative Innovation Processes", The XXVIII ISPIM Innovation Conference - Composing the Innovation Symphony, Vienna, Austria.

Simonsen, J. and Robertson, T. (2012), Routledge international handbook of participatory design, Public Understanding of Science, Routledge (Routledge international handbooks). http://dx.doi.org/10.1177/0963662512444848.

Timmermans, S. and Tavory, I. (2012), "Theory construction in qualitative research: From grounded theory to abductive analysis", Sociological Theory, Vol. 30 No. 3, pp. 167-186. http://dx.doi.org/10.1177/0735275112457914.

Topo, P. and Iltanen-Tähkävuori, S. (2010), "Scripting patienthood with patient clothing", Social Science and Medicine, Vol. 70 No. 11, pp. 1682-1689. http://dx.doi.org/10.1016/j.socscimed.2010.01.050.

Vinck, D. (2012), “Accessing Material Culture by Following Intermediary Objects”, In: L. Naidoo, (Ed.), An Ethnography of Global Landscapes and Corridors, INTECH Open Access Publisher, pp. 89-108. Available at: http://www.intechopen.com/books/an-ethnography-of-global-landscapes-and-corridors.

Wetter-Edman, K., Vink, J. and Blomkvist, J. (2017), "Staging aesthetic disruption through design methods for service innovation", Design Studies, Vol. 55 No. December, pp. 5-26. Elsevier Ltd, http://dx.doi.org/10.1016/j.destud.2017.11.007. 